\title{
Integration of functional neuronavigation and intraoperative MRI in surgery for drug-resistant extratemporal epilepsy close to eloquent brain areas
}

\author{
Björn Sommer, M.D., ${ }^{1}$ Peter Grummich, S.D., ${ }^{1}$ Roland Coras, M.D. ${ }^{3}$ \\ Burkhard Sebastian Kasper, M.D., ${ }^{2}$ Ingmar Blumcke, M.D., 3 \\ Hajo Martinus Hamer, M.D., ${ }^{2}$ Hermann Stefan, M.D., Ph.D., 2 \\ Michael Buchfelder, M.D., Ph.D., ${ }^{1}$ and Karl Roessler, M.D., Ph.D. ${ }^{1}$ \\ Departments of ${ }^{1}$ Neurosurgery, ${ }^{2}$ Neurology/Epilepsy Center, and ${ }^{3}$ Neuropathology, University Hospital \\ Erlangen, Germany
}

\begin{abstract}
Object. The authors performed a retrospective study to assess the impact of functional neuronavigation and intraoperative MRI (iMRI) on surgery of extratemporal epileptogenic lesions on postsurgical morbidity and seizure control.

Methods. Twenty-five patients (14 females and 11 males) underwent extratemporal resections for drug-resistant epilepsy close to speech/motor brain areas or adjacent to white matter tracts. The mean age at surgery was 34 years (range 12-67 years). The preoperative mean disease duration was 13.2 years. To avoid awake craniotomy, cortical motor-sensory representation was mapped during preoperative evaluation in 14 patients and speech representation was mapped in 15 patients using functional MRI. In addition, visualization of the pyramidal tract was performed in 11 patients, of the arcuate fascicle in 7 patients, and of the visual tract in 6 patients using diffusion tensor imaging. The mean minimum distance of tailored resection between the eloquent brain areas was $5.6 \mathrm{~mm}$. During surgery, blood oxygen level-dependent imaging and diffusion tensor imaging data were integrated into neuronavigation and displayed through the operating microscope. The postoperative mean follow-up was 44.2 months.

Results. In $20 \%$ of these patients, further intraoperative resection was performed because of intraoperatively documented residual lesions according to iMRI findings. At the end of resection, the final iMRI scans confirmed achievement of total resection of the putative epileptogenic lesion in all patients. Postoperatively, transient complications and permanent complications were observed in $20 \%$ and $12 \%$ of patients, respectively. Favorable postoperative seizure control (Engel Classes I and II) was achieved in $84 \%$ and seizure freedom in $72 \%$ of these consecutive surgical patients.

Conclusions. By using functional neuronavigation and iMRI for treatment of epileptogenic brain lesions, the authors achieved a maximum extent of resection despite the lesions' proximity to eloquent brain cortex and fiber tracts in all cases. The authors' results underline possible benefits of this technique leading to a favorable seizure outcome with acceptable neurological deficit rates in difficult-to-treat extratemporal epilepsy.

(http://thejns.org/doi/abs/10.3171/2013.2.FOCUS12397)
\end{abstract}

$\begin{array}{lllll}\text { KEY WORDS } & \text { functional neuronavigation } & \bullet & \text { eloquent brain area } & \bullet \\ \text { seizure outcome } & \bullet \quad \text { intraoperative MRI } & \text { extratemporal epilepsy } & \bullet & \text { surgery }\end{array}$

$\mathrm{E}$ XTRATEMPORAL epilepsy is one of the most challenging entities in the field of epilepsy surgery. Compared with temporal lobe epilepsy, where a good clinical outcome in terms of significant seizure reduction and improvement in quality of life after resection

\footnotetext{
Abbreviations used in this paper: $\mathrm{BOLD}=$ blood oxygen leveldependent; DNT $=$ dysembryoplastic neuroepithelial tumor; DTI = diffusion tensor imaging; $\mathrm{ECoG}=$ electrocorticography; $\mathrm{EEG}=$ electroencephalography; $\mathrm{FCD}=$ focal cortical dysplasia; fMRI = functional MRI; iMRI = intraoperative MRI; MEG = magnetoencephalography; MPRAGE = magnetization prepared rapid acquisition gradient echo.
}

has been reported to be between $58 \%$ and greater than $80 \%, 4,11,47,50$ extratemporal seizures are more difficult to treat surgically, with reported seizure freedom rates of only $10 \%-54 \% .9,16,24,27,52$

With the advent of iMRI, an intraoperative quality control was introduced to document whether a lesion had been resected sufficiently. ${ }^{18,38,41}$ Additionally, a dynamic adaption to different perioperative phenomena such as brain shift and localization of a residual lesion by updating navigation with intraoperative image data became possible. Moreover, damage of eloquent brain areas, such as motor, somatosensory, or speech cortex, can be avoided by integrating data from fMRI (BOLD imaging). 


\section{B. Sommer et al.}

Thus, resections adjacent to these important brain regions can be performed with more safety and efficiency. ${ }^{13,17,37}$ Additionally, white matter tracts can be detected ("fibertracking") by DTI and included in the neuronavigation, which we termed "multimodal navigation," 31 mainly to avoid awake craniotomies, which are cumbersome for the patient and surgeon.

Still, there are few studies that have reported on the short- and long-term outcomes of extratemporal lobe epilepsy surgery after integrating these technical advances. Thus, we retrospectively reviewed the records of patients with extratemporal lesions in or adjacent to eloquent brain who suffered from intractable epilepsy and who underwent epilepsy surgery at our center with the aid of integration of multimodal functional imaging and intraoperative high-field MRI.

\section{Methods}

\section{Examination Protocol}

From a total of 329 consecutive patients with treatment-refractory seizures who underwent surgery in our department between 2002 and 2011, 25 patients with extratemporal epilepsy were identified for this analysis. Inclusion criteria were 1) drug-resistant epilepsy, 2) extratemporal lesion location determined by MRI, 3) extratemporal epileptogenic focus, and 4) distance to eloquent brain area $\leq 20 \mathrm{~mm}$. Drug-resistant epilepsy had been defined according to the recent proposal by the International League Against Epilepsy. ${ }^{25}$ Each patient underwent an extensive presurgical epilepsy protocol ${ }^{45}$ that consisted of video-EEG monitoring, high-resolution (1.5- to 3-T) MRI, and neuropsychological testing. Additionally, 5 patients underwent invasive EEG monitoring with implantation of subdural and/or depth electrodes, and in 7 patients we obtained MEG recordings. Extratemporal localization of the epileptogenic zone was confirmed by these investigations at the Center of Epilepsy, University Hospital Erlangen. The results of these investigations were discussed in an interdisciplinary conference, for which a preliminary surgical treatment plan was established.

\section{Data Acquisition and Analysis}

BOLD Imaging. Functional MRI was performed using a 1.5-T MR clinical whole-body scanner with echo planar imaging (Magnetom Sonata, Siemens Medical Solutions) equipped with a standard head coil as previously described. ${ }^{14}$ A T1-weighted 3D data set (MPRAGE sequence; TE $4.38 \mathrm{msec}$, TR $2090 \mathrm{msec}$, matrix size 256 $\times 256$, FOV $256 \mathrm{~mm}$, slice thickness $1 \mathrm{~mm}$ ) was obtained. To localize eloquent brain areas, several stimulation paradigms were developed and tested. The tasks were chosen according to the individual cognitive abilities of each patient.

For the determination of the Broca and Wernicke cortical speech areas, patients had to perform specific tasks, including 1) performing the build a sentence from a noun task, 2) conjugating verbs, 3) answering questions, and 4) performing the picture-naming task. The activation protocols were described previously by Grummich and coworkers. ${ }^{14}$ The picture-naming task consisted of 40 animals and objects, which had to be named in random order. Furthermore, the sentence-reading task was used, where the introduction of grammatical patterns boosts language area activation in fMRI. Verbal memory performance was tested using a memorize numbers task. In this task, patients had to memorize specific words with each word standing for a character between the numbers 1 and 9. Then, the patients were tested on their ability to reproduce each number and the assigned word randomly. The arithmetic task was used both to test verbal memory and language areas, and patients were asked to either add up numbers one by one or build a sum.

DTI and Fiber Tracking. Diffusion tensor imaging makes use of the directional properties of diffusion of water molecules in brain tissue. The primary eigenvector from voxel to voxel is believed to represent the orientation of axons in white matter, which can be displayed as a neuronal fiber tract. For DTI, we applied a single-shot spin echo diffusion-weighted echo planar imaging sequence (TE 86 msec, TR 9200 msec, matrix size $128 \times 128$, FOV $198 \mathrm{~mm}$, slice thickness $1.9 \mathrm{~mm}$ ).

For reconstruction and visualization of the fiber tracts, we used the fiber-tracking module of the navigation planning software iPlan 2.6 (Brainlab). The process of localizing fiber bundles was described previously. 5,30 The starting point (region of interest) for pyramidal tract reconstruction depended on the lesion site and was chosen in the primary motor cortex (hand, arm, leg, and foot area). As a starting region of interest for the language and visual tracts, relevant brain areas such as the Wernicke and Broca area or primary visual cortex were chosen. The diffusion probability density function was used to determine diffusion tensors and their preferred direction within a specific 3D brain position (voxel). The proportion of molecules in a voxel was calculated by the vector from 6 different diffusion-weighted acquisitions, each obtained with a different orientation of the diffusion-sensitizing gradients. Here, we only used diffusion tensors, whose main axis of direction had a minimum fiber length of $50 \mathrm{~mm}$. After defining the mesencephalon as the target region, 3D tractography of the pyramidal tract was segmented. Tractography results were displayed in colorcoded fractional anisotropy maps (Fig. 1).

Manual segmentation of the epileptogenic lesion on the preoperative MR image and coregistration with functional imaging data were performed using iPlan 2.6 neuronavigation software (Brainlab AG). This data set was then fused with the first iMRI scan as described below. For determination of the least distance between eloquent brain areas, fiber tracts, and the epileptogenic lesion, we screened the appropriate MRI slices in every axis and measured the distance manually using the ruler function of the iPlan software. The mean minimal distance was $5.6 \mathrm{~mm}$.

Intraoperative MRI. All procedures were performed under general anesthesia. At the beginning of surgery, the patient's head was fixed in an MRI-compatible ceramic headholder. Then, the patient was moved from the operating position parallel to the scanner into the $1.5-\mathrm{T}$ iMRI unit (Magnetom Sonata Maestro Class, Siemens Health- 


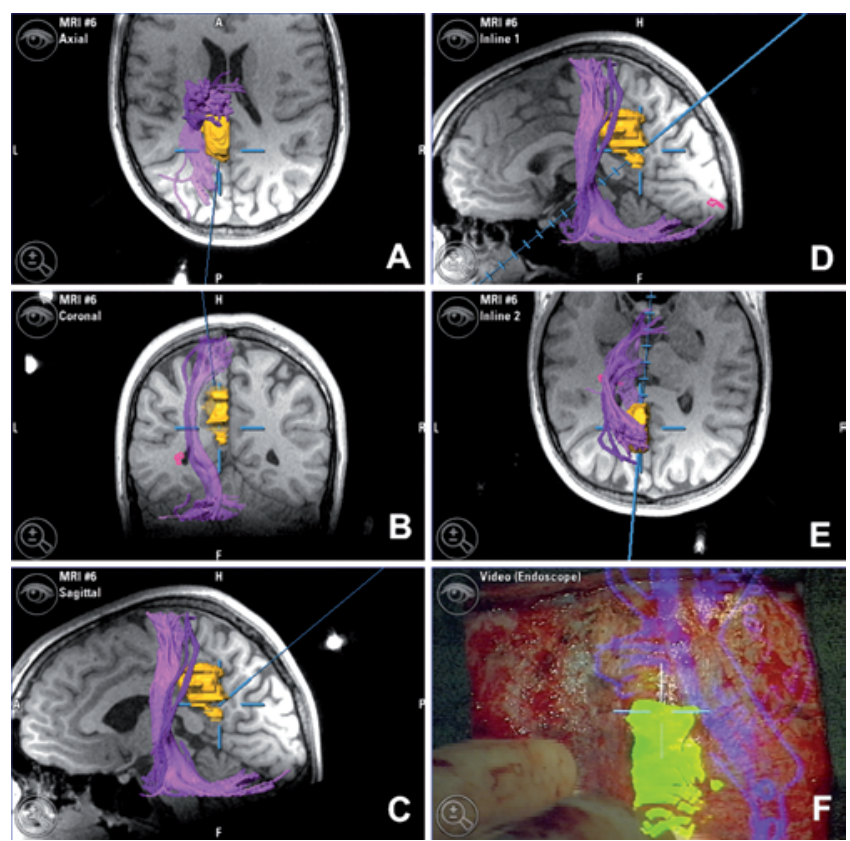

FIG. 1. Case 13. Lesionectomy performed for a DNT (WHO Grade I) in a 31-year-old woman. Initial axial (A and E), coronal (B), and sagittal (C and D) T1-weighted MPRAGE iMRI sequences showing the pyramidal tract (purple and pink tracts) and the segmented lesion (yellow cloud). The ideal trajectory and the target point (cross) are displayed in light blue. F: The intraoperative microscope view with projected neuronavigation data before dura opening.

care) to acquire the imaging data set. The intraoperative imaging sequences included a T1-weighted MPRAGE sequence (TE $4.38 \mathrm{msec}$, TR $2020 \mathrm{msec}$, matrix $128 \times 128$ [interpolated to $256 \times 256$ ], FOV $250 \mathrm{~mm}$, slice thickness $1 \mathrm{~mm}$, slab $16 \mathrm{~cm}$ ), T2-weighted coronal and transverse images (TE $98 \mathrm{msec}$, TR $6520 \mathrm{msec}$, matrix size $512 \times$ 307, FOV $250 \mathrm{~mm}$, slice thickness $3 \mathrm{~mm}$ ), and DTI sequences (TE $86 \mathrm{msec}$, TR $9200 \mathrm{msec}$, matrix size 128 $\times 128$, FOV $240 \mathrm{~mm}$, slice thickness $3 \mathrm{~mm}$ ). In cases of contrast-enhancing brain tumors, we administered Gddiethylenetriamine pentaacetic acid during intraoperative T1-weighted axial spin echo sequences for resection control. The pre- and intraoperative MRI sequences were acquired using the same protocol. Then, the patient was shifted back into the operating position. After fusion of preoperative functional data and iMRI data, the lesion was defined and manually segmented using our neuronavigation software (iPlan 2.6, Brainlab AG). Functional data were coregistered. Finally, data were transferred to an OPMI Pentero operation microscope (Zeiss), as described previously. ${ }^{29}$ The coregistration of iMRI with anatomical structures was accomplished with a mean error of $1.3 \pm 0.7 \mathrm{~mm}$ when surgery was started.

\section{Operative Technique}

We chose the surgical approach and type of resection according to the lesion site and the findings of the presurgical epilepsy protocol. A segmental resection entails removal of the circumscribed epileptogenic tissue with the perilesional cortex based on anatomical borders. In 2 patients, a "tailoring" of the resection included an extended lesion- ectomy with removal of the epileptogenic zone, which was defined in compliance with preoperative invasive EEG and MEG results as well as intraoperative ECoG with subdural strips and depth electrodes (Table 1). Microsurgical techniques were used for lesionectomies or tailored resections according to intraoperative functional navigation, where we kept close to the displayed boundaries of suspected pathological tissue and spared normal brain. After we had the impression of a complete resection, iMRI was performed. In cases of residual lesions, the patient was returned to the operative position, and after refreshing the neuronavigation with intraoperative data (Figs. 1 and 2), a complete resection was performed and confirmed by a second iMRI examination before the closing procedure.

\section{Definition of Postoperative Neurological Deterioration}

We documented neurological deterioration by careful clinical investigation before and immediately after surgery as well as 3 months after surgery and at the most recent follow-up visit for completing the follow-up data for this study. A severe neurological deficit included manifest hemiparesis, complete hemianopia, or hemineglect, which caused a clinically significant worsening in the patients' quality of life and led to disability and necessitated further medical care. Quadrantanopia, slight aphasia, and latent monoparesis were defined as mild deficits.

\section{Definition of Epilepsy Outcome}

We used the most recent Engel classification ${ }^{10}$ to evaluate postsurgery seizure outcome. An excellent outcome was defined as Engel Class IA, whereas a favorable outcome included Engel Classes I and II. Classes III and IV were considered poor outcome.
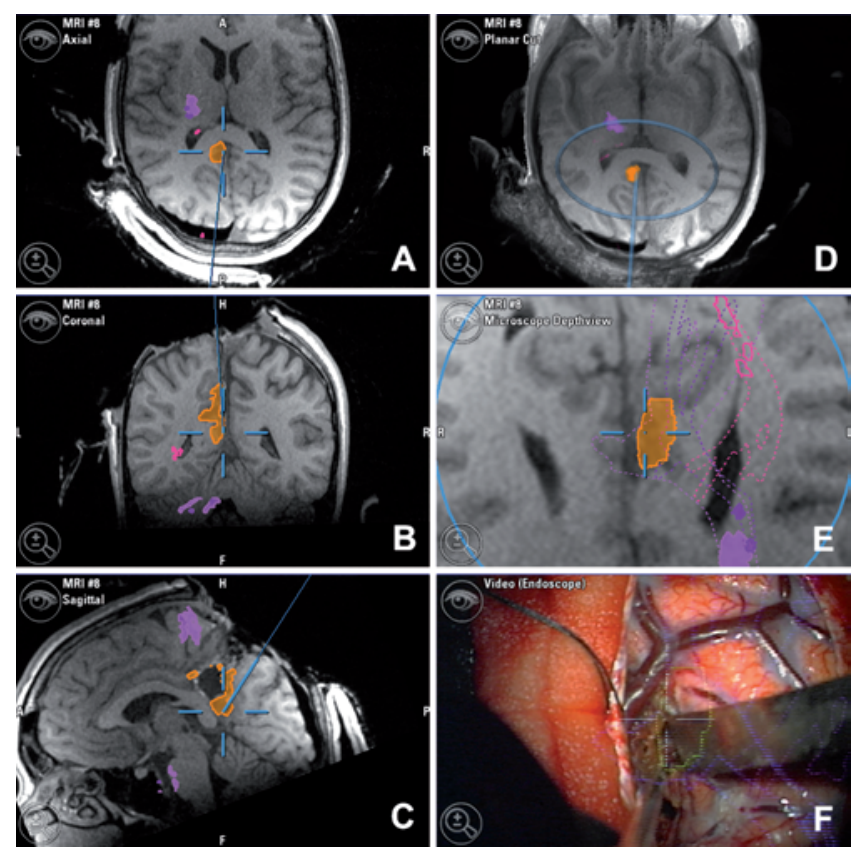

FIG. 2. Case 13. Intraoperative MR images. After resection, the control iMRI showed a remnant tumor mass. Neuronavigational data were updated, and the remaining lesion (orange) was manually segmented $(\mathrm{A}-\mathrm{E})$. The microscope view of the resection cavity and the segmented lesion are depicted in panel $\mathbf{F}$. 


\section{B. Sommer et al.}

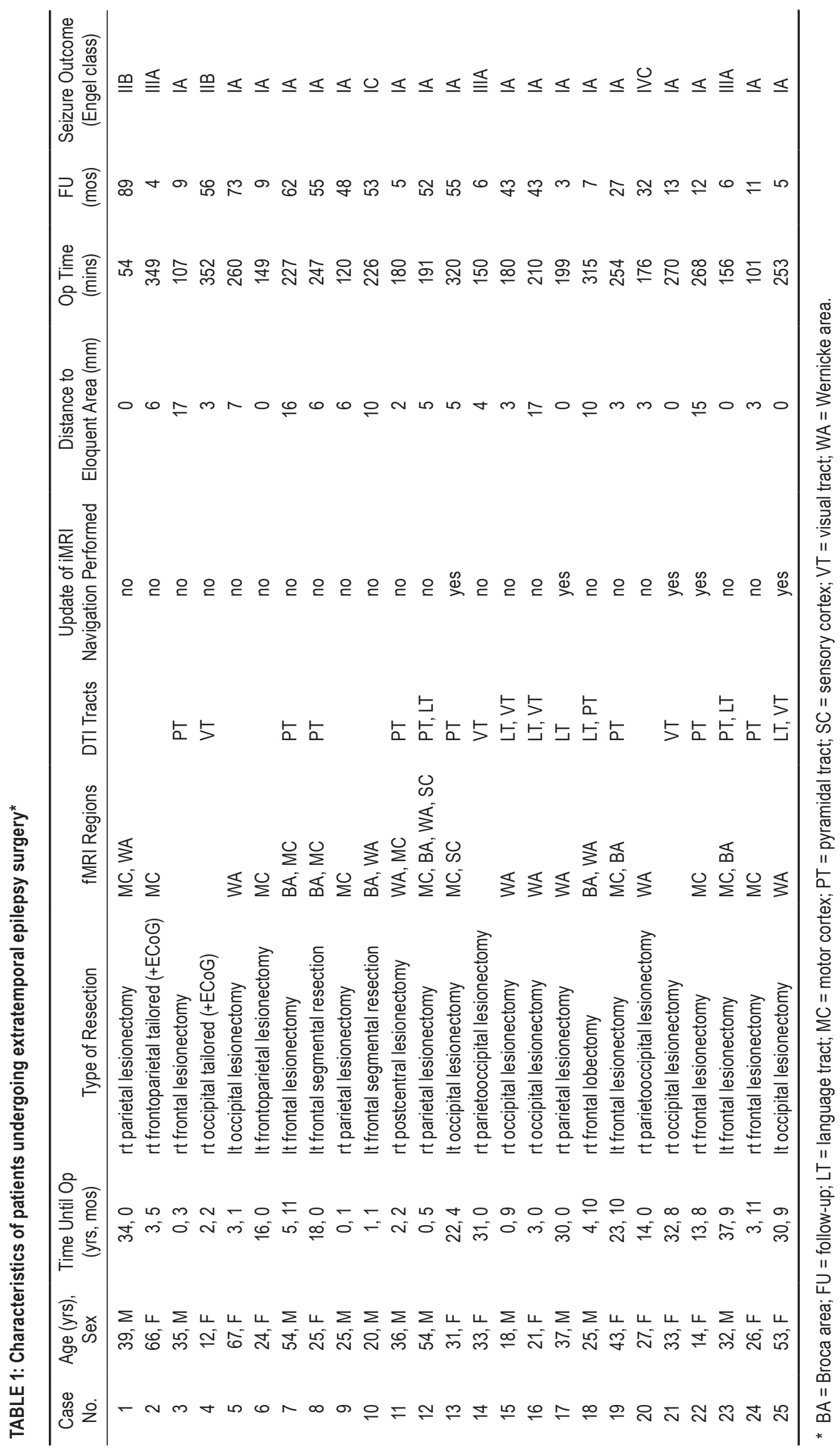




\section{Multimodal neuronavigation in extratemporal epilepsy surgery}

Patients' most recent neurological and epilepsy outcome data were obtained from the follow-up examinations in the Neurological Epilepsy Center at the University Hospital Erlangen and via telephone interviews.

\section{Results}

Twenty-five patients (14 females and 11 males) with drug-resistant epilepsy underwent presurgical evaluation and epilepsy surgery between September 2002 and November 2011 at the University Hospital of Erlangen. Demographic and clinical data of the patients are presented in Table 1. Nine resections were performed in the left hemisphere. The mean preoperative duration of pharmacoresistant epilepsy was 13.2 years (range 0-34 years). Patients underwent follow-up for a mean of $44.2 \pm 26.9$ months after surgery.

\section{Neuroimaging Findings}

Preoperative MRI identified a structural lesion corresponding to the epileptogenic zone in all patients. The distribution of the pathological tissue was seen in the following brain regions: frontal $(\mathrm{n}=9)$, frontoparietal $(\mathrm{n}=$ $2)$, parietal $(n=5)$, parietooccipital $(n=2)$, and occipital $(\mathrm{n}=7)$.

\section{Amount of Resection and Histology}

In all cases, complete lesionectomy or cortical resection according to functional data was documented by iMRI. Table 1 illustrates that a complete resection of the predefined lesion was confirmed intraoperatively in 20 patients (80\%). In 5 patients (Cases 13, 17, 21, 22, and 25), resection was considered as incomplete and an update of neuronavigation was performed (Fig. 2). A complete resection then was achieved by extending the surgery, as confirmed by iMRI. The mean operating time was $213.3 \pm$ 78.7 minutes, while overall scan time for the acquisition of intraoperative MRI sequences (T1-weighted MPRAGE, T2-weighted coronal and axial, DTI) was 13.9 minutes.

Histopathological examination of resected tissue revealed FCDs in 7 patients (1 Type IIa FCD, 6 Type IIb FCDs according to the classification of Blümcke et al. ${ }^{3}$ ), posttraumatic glial scars in 5, cavernous hemangiomas in 5 , DNTs in 2, anaplastic astrocytomas in 2, gangliogliomas in 2 , neocortex with reactive gliosis in 1 , and vascular hamartoma in 1 patient.

\section{Neurological Outcome}

Mild neurological deterioration was seen in 2 patients: the patient in Case 4 who had previously undergone surgery for meningioma and who underwent resection of postsurgical occipital lobe gliosis, and the patient in Case 16 who underwent a hemangioma resection. These patients suffered from a quadrantanopia despite intraoperative integration of functional (visual tract) neuronavigation. Overall, 4 patients experienced a transient dysphasia: in 1 patient (Case 5, left occipital lobe gliosis), the preexisting Wernicke aphasia was aggravated for the duration of 6 postoperative days, the patient in Case 7 experienced a transient Broca aphasia for 3 days after resection of a left frontal anaplastic astrocytoma, and the patient in Case 23 with a left frontal Type IIb FCD reported amnestic aphasia lasting 5 days. In 1 patient (Case 13, who had a left occipital DNT), transient amnestic aphasia lasted as long as 6 months. In 3 of those 4 patients, language mapping had been used except for the patient in Case 13, in whom the lesion was located far away from speech areas (Table 1).

Another patient (Case 22 with a right frontal Type IIb FCD), in whom we preoperatively segmented the motor cortex and the pyramidal tract, experienced a transient paresis of the left arm lasting the duration of the 1st postoperative day (Fig. 3). Severe surgical deficits occurred in 1 patient (Case 6), in whom the lesion was located within the motor cortex and who experienced a permanent hemiparesis postoperatively, from which the patient fortunately recovered almost completely after 9 months. Overall, $20 \%$ of all patients in this study had a transient, $8 \%$ a permanent minor, and $4 \%$ a severe permanent neurological deficit (Table 2).

\section{Seizure Outcome}

Of all patients, $18(72 \%)$ of 25 had an excellent outcome and were completely seizure free. Favorable seizure outcome was achieved in 21 (84\%) of 25 patients (Fig. 4). In $16 \%$, we found a poor outcome with 1 patient (Case 20) who had an aggravation of seizure frequency and intensity (Engel Class IVC).

Four-year follow-up data were available in 13 patients, of whom $11(85 \%)$ had Engel Class I or II seizure outcome. Of all patients who had either transient (4 patients, Engel Classes IA, IA, IA, and IIIA) or permanent (3 patients, Engel Classes IA, IA, and IIB) neurological deficits, $71 \%$ were seizure free.

\section{Postsurgical Complications}

Among the 25 neurosurgical interventions, there was
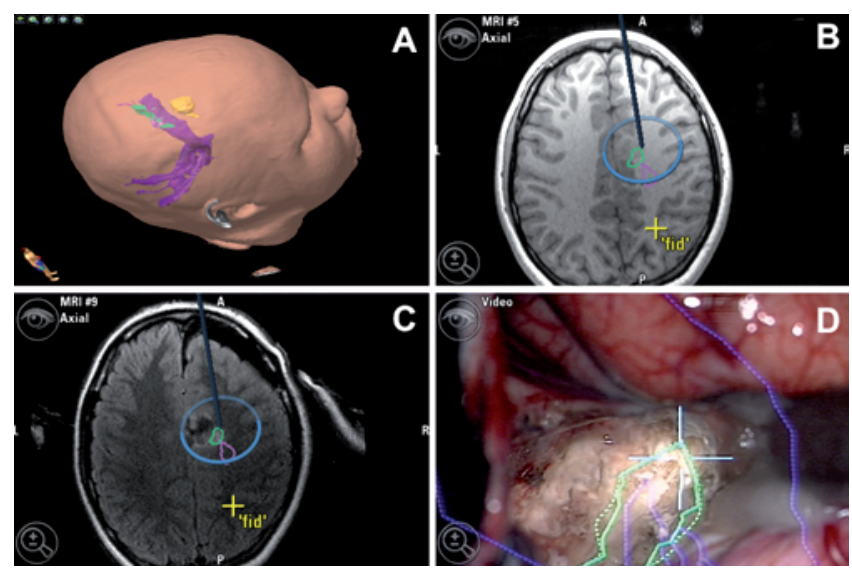

FIG. 3. Case 22. Example of a patient with transient monoparesis of the left arm after resection of a Type Ilb FCD. Preoperative 3D view of functional data $(A)$ and $\mathrm{MRI}(\mathrm{B})$ before resection showing the localization of the lesion (yellow) in relation to the motor cortex (light green) and pyramidal tract (purple). Note the resegmentation of the remnant lesion (light green) and its vicinity to the pyramidal tract (purple) on the iMRI control scan (C) and the corresponding microscope view (D) including projected navigation data. 'fid' denotes the position of the scalp fiducial for additional registration accuracy control. 
TABLE 2: Properties of fMRI and DTI

\begin{tabular}{lclc}
\hline & \multicolumn{2}{c}{$\begin{array}{c}\text { Neurological Deficit After } \\
\text { Epilepsy Surgery }\end{array}$} \\
\cline { 3 - 4 } \multicolumn{1}{c}{ Imaging Modality } & $\begin{array}{c}\text { No. of } \\
\text { Patients }\end{array}$ & Transient & Permanent \\
\hline functional imaging & & & \\
MC & 13 & $1 / 13$ & $1 / 13$ \\
SC & 2 & 0 & 0 \\
BA & 7 & $2 / 7$ & 0 \\
WA & 11 & $2 / 11$ & 0 \\
language (BA+WA) & 3 & 0 & 0 \\
DTI & & & \\
PT & 11 & $1 / 11$ & 0 \\
LT & 7 & 0 & 0 \\
VT & 6 & 0 & $2 / 6$ \\
\hline
\end{tabular}

1 wound infection (4\%), which led to a local wound revision. No severe surgical complications such as intracranial hemorrhage, meningitis, or death occurred.

\section{Discussion}

Our retrospective analysis demonstrates that in patients with lesional extratemporal epilepsy, multimodal navigation assists in the decision making of whether resection is justified, can offer patients with pharmacoresistant epilepsy a good chance to gain seizure control with acceptable risks, can reduce the rate of incomplete resections when used in combination with iMRI, and can be implemented in daily routine surgery at epilepsy centers.

\section{Lesional Extratemporal Epilepsy Surgery}

There are different reasons why extratemporal lesionectomies only represent a small percentage of about $9 \%$ $18 \%$ of all epilepsy surgeries. ${ }^{2,4}$ First, extratemporal lobe lesions present with a variety of histopathological findings, ${ }^{12}$ which are unsteady and diffuse and not that well defined as, for example, hippocampal sclerosis. Some of them, such as cortical dysplasia, turn out to have a more favorable course and chance of surgical success. ${ }^{9,40}$ However, even with additional diagnostic tools such as MEG,

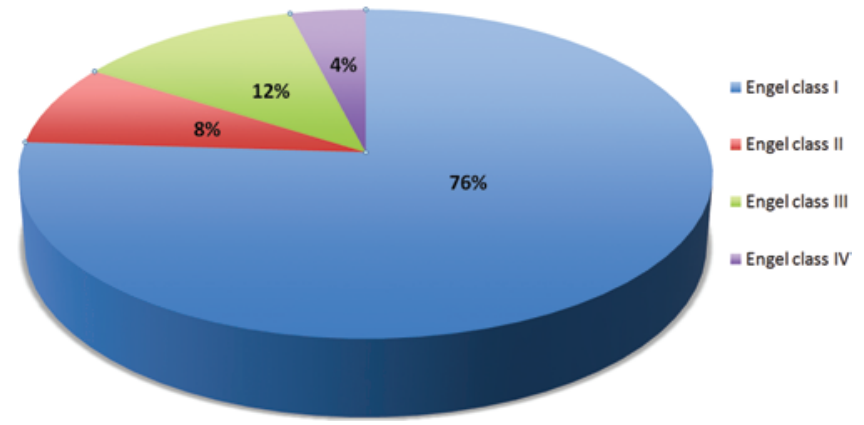

FIG. 4. Pie chart showing the seizure outcome at last follow-up according to Engel classification. Seventy-two percent of all patients (18 of 25) had excellent (Engel IA); 84\% (21) had favorable; and 16\% (4) had poor seizure control. intraoperative electrocorticography, or invasive electrode grid implantation, the exact anatomical localization of the epileptogenic zone often remains inaccurate. Finally, the localization of extratemporal lesions in the vicinity of functional cortex or fiber tracts make them difficult to resect without harming susceptible brain tissue and causing severe permanent neurological deficits. Our patients had mainly well-defined epileptogenic lesions, such as FCDs, intrinsic WHO Grade I tumors, or vascular lesions. However, 24\% of our patients suffered from ill-defined epileptic foci, where histological investigation displayed gliotic tissue and another $8 \%$ had diffuse gliomas. In a subgroup analysis of patients (Cases 1, 4, 6, 11, 14, 15, 17, 19-21, and 23-25) in whom the minimum distance between the lesion and eloquent brain structures lay between 0 and $4 \mathrm{~mm}$ (Table 1 ), we noticed only 2 patients with a permanent neurological deficit (quadrantanopia in Case 4 and hemiparesis in Case 6). It becomes apparent that in epileptogenic foci within or very close to eloquent brain areas, epilepsy surgery is still possible with the aid of multimodal navigation and intraoperative imaging.

\section{Functional MRI, Tractography (DTI), Neuronavigation, and $i M R I$}

Extratemporal surgery of lesions close to eloquent brain areas needs sophisticated processing of image data compared with standard temporal lobe surgery. BOLD MRI and DTI tractography are noninvasive, useful tools to detect eloquent gray matter and white matter tracts. Their accuracy and specificity were discussed in recent studies. ${ }^{1,28,36,37}$ Eloquent cortex that can be mapped using fMRI includes the primary motor and somatosensory regions, and Broca and Wernicke areas along with hemispheric language lateralization and their connectivity within the CNS by using DTI for displaying pyramidal, language, and visual tracts. ${ }^{32,51}$

Intraoperative high-field ( $\geq 1.5-\mathrm{T}$ ) MRI systems are well established in operating rooms and were used in glioma surgery or frameless stereotaxy. ${ }^{23,41}$ To our knowledge, only a few studies on epilepsy surgery performed with the aid of 1.5-T iMRI systems exist. ${ }^{5,21,22,26,46} \mathrm{Neu}-$ ronavigation became popular recently for lesionectomy of temporal and extratemporal regions, ${ }^{6}$ lesions of the insular region, ${ }^{49}$ and callosotomy. ${ }^{20}$ With more than 15 years of experience with neuronavigation and intraoperative imaging, our department successfully developed and integrated multimodal imaging techniques in daily neurosurgery.

In our retrospective investigation of patients with lesional extratemporal epilepsy, fMRI and DTI tractography, as well as their combination with iMRI, were successfully implemented in routine resective epilepsy procedures. This multimodal approach led to a complete resection of the lesion in all 25 of our patients, which seems an exceptional result, compared with the published incomplete extratemporal lesionectomy resection rates ranging from $71 \%$ to $85 \%$ (Table 3 ). The observation that the neurological outcome is significantly improved when compared with previously published studies ${ }^{2,4,40}$ stresses the application of functional navigation and iMRI as an advantageous technique. 
Multimodal neuronavigation in extratemporal epilepsy surgery

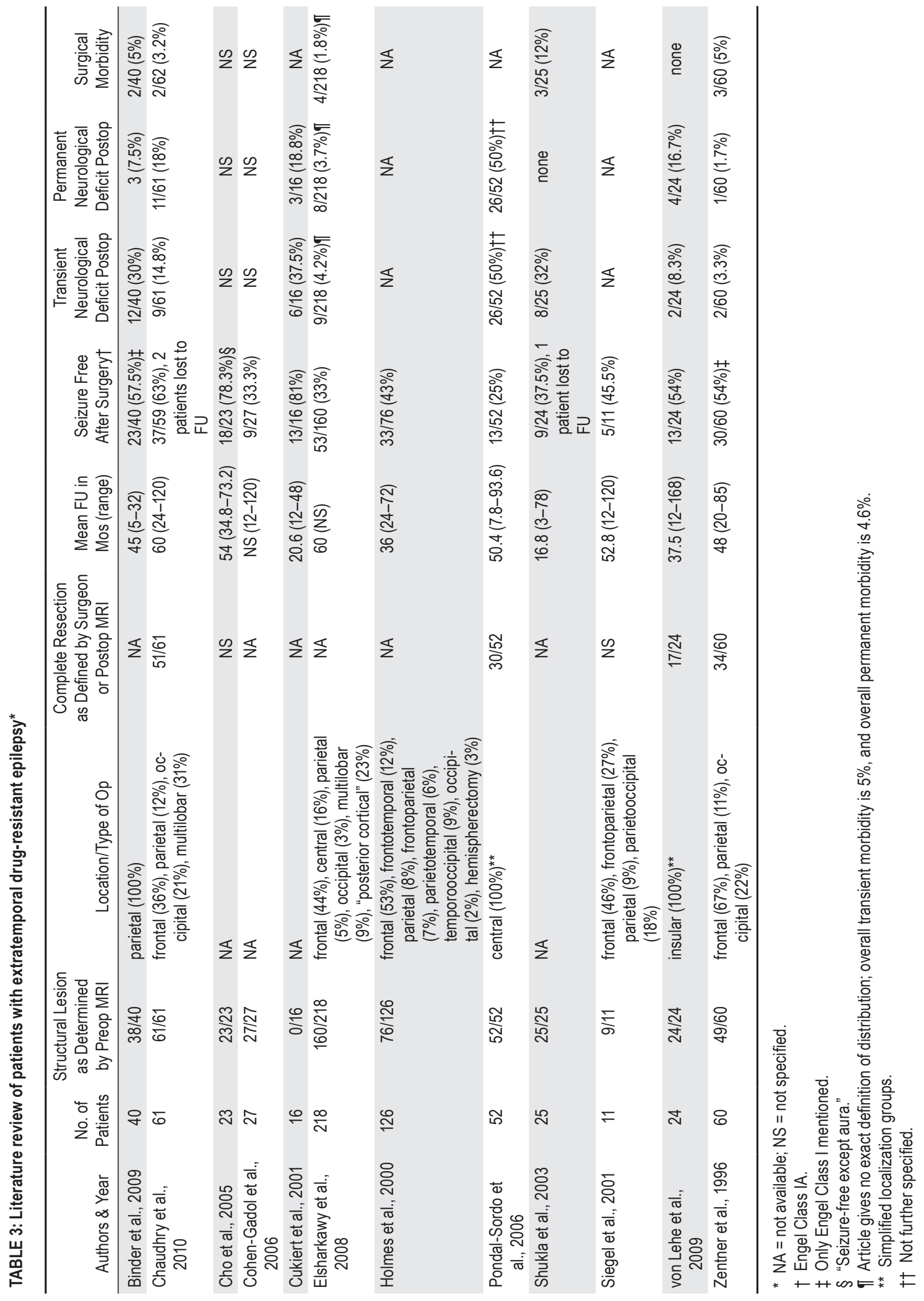




\section{B. Sommer et al.}

Awake craniotomy with intraoperative cortical brain mapping is considered to be the primary method to monitor eloquent brain areas during surgery. ${ }^{42}$ However, the superiority of this technique regarding efficiency and safety is still a matter of debate, lacking randomized controlled trials with larger patient cohorts. ${ }^{15,33}$ Inadequate pain control, airway obstruction leading to intubation, vomiting, brain swelling, and intraoperative seizures are among the common objectionable events during awake surgery. The risk of new appearance or worsening of permanent neurological deficits using this technique has been reported to be between $0 \%$ and $29 \%$, with surgical complication rates between $0 \%$ and $14.8 \% .^{15,33,42}$ Regarding intraoperative electrophysiological monitoring, we based our strategy on previously published results in 230 patients from our department in which we compared cortical brain mapping and functional neuronavigation to identify the sensorimotor cortex. ${ }^{39}$

Considering the aforementioned obstacles of awake craniotomy from our own experience, as well as the accompanying promising results, $, 5,29,30$ we preferred a noninvasive preoperative localization of eloquent brain areas by fMRI and DTI with general anesthesia over awake craniotomy and found no inferiority regarding neurological outcome and surgical complication rates.

\section{Neurological and Seizure Outcome}

There is evidence that in the presence of brain lesions, patients undergoing extratemporal surgery have better epilepsy control than those without lesions..$^{19,24,40}$ In terms of neurological outcome, our results are even more favorable than those in other studies, where permanent deficits occurred in up to $18 \%$ of all lesional extratemporal epilepsy operations (Table 3). One patient (4\%) suffered from a hemiparesis after resection of an FCD that was located immediate to the primary motor cortex. Fortunately, the patient recovered almost completely after 9 months. Two more patients with a postsurgical occipital gliosis and a cavernoma near the visual field tracts had quadrantanopia, which is below the reported incidence of visual field defects after anterior temporal lobe resections. ${ }^{35}$

In our study, favorable seizure outcome in lesional extratemporal lobe epilepsy (Engel Classes I and II) was achieved in $84 \%$ of all patients, of whom $72 \%$ are completely seizure free after resection of an epileptogenic lesion near eloquent cortex (Engel Class IA). The proximity to eloquent brain areas with a mean minimum distance to the lesion of $5.6 \mathrm{~mm}$ and the long follow-up period of nearly 4 years on average highlight the favorable results of our study, which were achieved using advanced multimodal neuronavigation procedures. However, we cannot give a reliable safety margin in terms of absolute values to avoid damage to eloquent structures. The errors that occur during calculation of functional data, target registration of the navigation system, coregistration while fusing the pre- and intraoperative MR images, and the observer error in the manual segmentation procedure are cumulative and leave no room for an exact threshold determination in our series.

Studies on extratemporal lesionectomy reported seizure-free (Engel Class I) rates ranging between $25 \%$ and
$57.5 \%$ (Table 3). A meta-analysis by Téllez-Zenteno and colleagues ${ }^{48}$ identified 6 studies regarding extratemporal epilepsy (4 in children and 2 in adults) between 1995 and 2007, which showed a seizure-free outcome of 53\% (lesional, 95\% CI 28\%-68\%) and 26\% (nonlesional, 95\% CI $13 \%-38 \%$ ) for all patients. Thus, our results are remarkable compared with recent literature and previously published series of extratemporal epilepsy surgery.

\section{Limitations of This Retrospective Investigation}

Important limitations of our study are the lack of prospective, randomized controlled data that compare surgery of extratemporal lesions close to eloquent cortical structures with and without using multimodal neuronavigation. Another drawback of this study is the small sample size. Further improvement concerning the identification of eloquent brain areas and possible postsurgical deficits could have been made performing awake craniotomies and using intraoperative electrophysiological and neurological monitoring. Compared with other observations and their presurgical diagnostic workup, we demonstrate a consequent integration of functional data and iMRI in all investigated patients. The acquisition of iMRI sequences only minimally increases the operating time. This is tolerated in favor of a remarkable outcome in our series, and no complications occurred due to a longer operating time. In 5 of 25 patients, the course of surgery was corrected according to iMRI results, which led to complete lesionectomy or cortical resection. Additionally, questions arose concerning the usefulness of surgical planning and accuracy of the noninvasive fMRI method. For example, the relatively gross spatial solution of fMRI studies and the threshold rate of fMRI data can lead to inaccuracy in localization and interpretation of BOLD signal intensity and activation patterns. Another problem concerning the use of preoperative functional data during surgery is volumetric brain deformation and intraoperative loss of cerebrospinal fluid ("brain shift"), which is known to diminish the accuracy of the depicted eloquent brain areas and fiber tracts. In our study, we took into account the brain structure translocation and performed an intraoperative MRI update in 5 patients with new acquisition of navigation data and reassignment of residual pathological tissue using the navigation planning software. In all of those patients, remnant tissue could be removed completely, and fortunately, all were seizure free and only 1 patient suffered from discrete amnestic aphasia.

\section{Conclusions}

In extratemporal pharmacoresistant lesional epilepsy, functional neuronavigation and iMRI are useful tools for performing resections close to eloquent brain areas. Our study supports the estimation of maximum resection extent independently of the distance to important functional brain areas and guides through the operation. These favorable results demonstrate that intraoperative functional neuronavigation is a major contribution to the prevention of functional deficits in presumably unresectable lesions, where in the past the risk-benefit ratio was more unfavorable for the patient. Considering possible brain shift 


\section{Multimodal neuronavigation in extratemporal epilepsy surgery}

during the surgery, iMRI can adjust inaccuracy of coregistration and improve the accuracy of functional mapping and fiber tracking. This method may lead to avoidance or marked reduction of postoperative neurological deficits.

\section{Disclosure}

The authors report no conflict of interest concerning the materials or methods used in this study or the findings specified in this paper.

Author contributions to the study and manuscript preparation include the following. Conception and design: Sommer, Buchfelder, Roessler. Acquisition of data: Sommer, Grummich, Coras, Kasper. Analysis and interpretation of data: Sommer, Grummich, Kasper, Roessler. Drafting the article: Sommer, Roessler. Critically revising the article: Grummich, Coras, Kasper, Blumcke, Hamer, Stefan, Buchfelder, Roessler. Reviewed submitted version of manuscript: Sommer, Roessler. Approved the final version of the manuscript on behalf of all authors: Sommer. Study supervision: Roessler.

\section{References}

1. Bartos R, Jech R, Vymazal J, Petrovický P, Vachata P, Hejcl A, et al: Validity of primary motor area localization with fMRI versus electric cortical stimulation: a comparative study. Acta Neurochir (Wien) 151:1071-1080, 2009

2. Binder DK, Podlogar M, Clusmann H, Bien C, Urbach H, Schramm J, et al: Surgical treatment of parietal lobe epilepsy. Clinical article. J Neurosurg 110:1170-1178, 2009

3. Blümcke I, Thom M, Aronica E, Armstrong DD, Vinters HV, Palmini A, et al: The clinicopathologic spectrum of focal cortical dysplasias: a consensus classification proposed by an ad hoc Task Force of the ILAE Diagnostic Methods Commission. Epilepsia 52:158-174, 2011

4. Chaudhry N, Radhakrishnan A, Abraham M, Kesavadas C, Radhakrishnan VV, Sankara Sarma P, et al: Selection of ideal candidates for extratemporal resective epilepsy surgery in a country with limited resources. Epileptic Disord 12:38-47, 2010

5. Chen X, Weigel D, Ganslandt O, Buchfelder M, Nimsky C: Prediction of visual field deficits by diffusion tensor imaging in temporal lobe epilepsy surgery. Neuroimage 45:286-297, 2009

6. Cho DY, Lee WY, Lee HC, Chen CC, Tso M: Application of neuronavigator coupled with an operative microscope and electrocorticography in epilepsy surgery. Surg Neurol 64:411-418, 2005

7. Cohen-Gadol AA, Wilhelmi BG, Collignon F, White JB, Britton JW, Cambier DM, et al: Long-term outcome of epilepsy surgery among 399 patients with nonlesional seizure foci including mesial temporal lobe sclerosis. J Neurosurg 104:513-524, 2006

8. Cukiert A, Buratini JA, Machado E, Sousa A, Vieira JO, Argentoni M, et al: Results of surgery in patients with refractory extratemporal epilepsy with normal or nonlocalizing magnetic resonance findings investigated with subdural grids. Epilepsia 42:889-894, 2001

9. Elsharkawy AE, Pannek H, Schulz R, Hoppe M, Pahs G, Gyimesi $\mathrm{C}$, et al: Outcome of extratemporal epilepsy surgery experience of a single center. Neurosurgery 63:516-526, 2008

10. Engel J, Van Ness PC, Rasmussen TB, Ojemann LM: Outcome with respect to epileptic seizures, in Engel J (ed): Surgical Treatment of the Epilepsies, ed 2. New York: Raven Press, 1993, pp 609-621

11. Engel J Jr, Wiebe S, French J, Sperling M, Williamson P, Spencer D, et al: Practice parameter: temporal lobe and localized neocortical resections for epilepsy. Epilepsia 44:741-751, 2003

12. Frater JL, Prayson RA, Morris HH III, Bingaman WE: Sur- gical pathologic findings of extratemporal-based intractable epilepsy: a study of 133 consecutive resections. Arch Pathol Lab Med 124:545-549, 2000

13. Gasser T, Szelenyi A, Senft C, Muragaki Y, Sandalcioglu IE, Sure U, et al: Intraoperative MRI and functional mapping. Acta Neurochir Suppl 109:61-65, 2011

14. Grummich P, Nimsky C, Pauli E, Buchfelder M, Ganslandt O: Combining fMRI and MEG increases the reliability of presurgical language localization: a clinical study on the difference between and congruence of both modalities. Neuroimage 32:1793-1803, 2006

15. Gupta DK, Chandra PS, Ojha BK, Sharma BS, Mahapatra AK, Mehta VS: Awake craniotomy versus surgery under general anesthesia for resection of intrinsic lesions of eloquent cortex - a prospective randomised study. Clin Neurol Neurosurg 109:335-343, 2007

16. Haglund MM, Ojemann GA: Extratemporal resective surgery for epilepsy. Neurosurg Clin N Am 4:283-292, 1993

17. Hall WA, Kim P, Truwit CL: Functional magnetic resonance imaging-guided brain tumor resection. Top Magn Reson Imaging 19:205-212, 2009

18. Hall WA, Truwit CL: Intraoperative MR-guided neurosurgery. J Magn Reson Imaging 27:368-375, 2008

19. Holmes MD, Kutsy RL, Ojemann GA, Wilensky AJ, Ojemann LM: Interictal, unifocal spikes in refractory extratemporal epilepsy predict ictal origin and postsurgical outcome. Clin Neurophysiol 111:1802-1808, 2000

20. Jea A, Vachhrajani S, Johnson KK, Rutka JT: Corpus callosotomy in children with intractable epilepsy using frameless stereotactic neuronavigation: 12-year experience at the Hospital for Sick Children in Toronto. Neurosurg Focus 25(3):E7, 2008

21. Kaibara T, Myles ST, Lee MA, Sutherland GR: Optimizing epilepsy surgery with intraoperative MR imaging. Epilepsia 43:425-429, 2002

22. Kelly JJ, Hader WJ, Myles ST, Sutherland GR: Epilepsy surgery with intraoperative MRI at 1.5 T. Neurosurg Clin N Am 16:173-183, 2005

23. Kuhnt D, Ganslandt O, Schlaffer SM, Buchfelder M, Nimsky C: Quantification of glioma removal by intraoperative highfield magnetic resonance imaging: an update. Neurosurgery 69:852-863, 2011

24. Kutsy RL: Focal extratemporal epilepsy: clinical features, EEG patterns, and surgical approach. J Neurol Sci 166:1-15, 1999

25. Kwan P, Arzimanoglou A, Berg AT, Brodie MJ, Allen Hauser W, Mathern G, et al: Definition of drug resistant epilepsy: consensus proposal by the ad hoc Task Force of the ILAE Commission on Therapeutic Strategies. Epilepsia 51:1069-1077, 2010

26. Levy R, Cox RG, Hader WJ, Myles T, Sutherland GR, Hamilton MG: Application of intraoperative high-field magnetic resonance imaging in pediatric neurosurgery. Clinical article. J Neurosurg Pediatr 4:467-474, 2009

27. McIntosh AM, Averill CA, Kalnins RM, Mitchell LA, Fabinyi GC, Jackson GD, et al: Long-term seizure outcome and risk factors for recurrence after extratemporal epilepsy surgery. Epilepsia 53:970-978, 2012

28. Mehta AD, Klein G: Clinical utility of functional magnetic resonance imaging for brain mapping in epilepsy surgery. Epilepsy Res 89:126-132, 2010

29. Nimsky C, Ganslandt O, Merhof D, Sorensen AG, Fahlbusch $\mathrm{R}$ : Intraoperative visualization of the pyramidal tract by diffusion-tensor-imaging-based fiber tracking. Neuroimage 30: 1219-1229, 2006

30. Nimsky C, Grummich P, Sorensen AG, Fahlbusch R, Ganslandt O: Visualization of the pyramidal tract in glioma surgery by integrating diffusion tensor imaging in functional neuronavigation. Zentralbl Neurochir 66:133-141, 2005

31. Nimsky C, Kuhnt D, Ganslandt O, Buchfelder M: Multimodal 


\section{B. Sommer et al.}

navigation integrated with imaging. Acta Neurochir Suppl 109:207-214, 2011

32. O'Shea JP, Whalen S, Branco DM, Petrovich NM, Knierim KE, Golby AJ: Integrated image- and function-guided surgery in eloquent cortex: a technique report. Int J Med Robot 2:75-83, 2006

33. Pereira LC, Oliveira KM, L'Abbate GL, Sugai R, Ferreira JA, da Motta LA: Outcome of fully awake craniotomy for lesions near the eloquent cortex: analysis of a prospective surgical series of 79 supratentorial primary brain tumors with long follow-up. Acta Neurochir (Wien) 151:1215-1230, 2009

34. Pondal-Sordo M, Diosy D, Téllez-Zenteno JF, Girvin JP, Wiebe S: Epilepsy surgery involving the sensory-motor cortex. Brain 129:3307-3314, 2006

35. Powell HW, Parker GJ, Alexander DC, Symms MR, Boulby PA, Wheeler-Kingshott CA, et al: MR tractography predicts visual field defects following temporal lobe resection. Neurology 65:596-599, 2005

36. Richardson M: Current themes in neuroimaging of epilepsy: brain networks, dynamic phenomena, and clinical relevance. Clin Neurophysiol 121:1153-1175, 2010

37. Roessler K, Donat M, Lanzenberger R, Novak K, Geissler A, Gartus A, et al: Evaluation of preoperative high magnetic field motor functional MRI (3 Tesla) in glioma patients by navigated electrocortical stimulation and postoperative outcome. J Neurol Neurosurg Psychiatry 76:1152-1157, 2005

38. Roessler K, Ungersboeck K, Aichholzer M, Dietrich W, Goerzer H, Matula C, et al: Frameless stereotactic lesion contourguided surgery using a computer-navigated microscope. Surg Neurol 49:282-289, 1998

39. Romstöck J, Fahlbusch R, Ganslandt O, Nimsky C, Strauss $\mathrm{C}$ : Localisation of the sensorimotor cortex during surgery for brain tumours: feasibility and waveform patterns of somatosensory evoked potentials. J Neurol Neurosurg Psychiatry 72: 221-229, 2002

40. Roper SN: Surgical treatment of the extratemporal epilepsies. Epilepsia 50 (Suppl 8):69-74, 2009

41. Senft C, Bink A, Franz K, Vatter H, Gasser T, Seifert V: Intraoperative MRI guidance and extent of resection in glioma surgery: a randomised, controlled trial. Lancet Oncol 12:997_ 1003, 2011

42. Serletis D, Bernstein M: Prospective study of awake craniotomy used routinely and nonselectively for supratentorial tumors. J Neurosurg 107:1-6, 2007

43. Shukla G, Bhatia M, Singh VP, Jaiswal A, Tripathi M, Gaik- wad S, et al: Successful selection of patients with intractable extratemporal epilepsy using non-invasive investigations. Seizure 12:573-576, 2003

44. Siegel AM, Jobst BC, Thadani VM, Rhodes CH, Lewis PJ, Roberts DW, et al: Medically intractable, localization-related epilepsy with normal MRI: presurgical evaluation and surgical outcome in 43 patients. Epilepsia 42:883-888, 2001

45. Stefan H, Buchfelder M: Presurgical evaluation for epilepsy surgery. Neurol Psych Brain Res 7:109-112, 1999

46. Sun GC, Chen XL, Zhao Y, Wang F, Song ZJ, Wang YB, et al: Intraoperative MRI with integrated functional neuronavigation-guided resection of supratentorial cavernous malformations in eloquent brain areas. J Clin Neurosci 18:1350-1354, 2011

47. Téllez-Zenteno JF, Dhar R, Wiebe S: Long-term seizure outcomes following epilepsy surgery: a systematic review and meta-analysis. Brain 128:1188-1198, 2005

48. Téllez-Zenteno JF, Hernández Ronquillo LH, Moien-Afshari F, Wiebe S: Surgical outcomes in lesional and non-lesional epilepsy: a systematic review and meta-analysis. Epilepsy Res 89:310-318, 2010

49. von Lehe M, Wellmer J, Urbach H, Schramm J, Elger CE, Clusmann H: Insular lesionectomy for refractory epilepsy: management and outcome. Brain 132:1048-1056, 2009

50. Wiebe S, Blume WT, Girvin JP, Eliasziw M: A randomized, controlled trial of surgery for temporal-lobe epilepsy. N Engl J Med 345:311-318, 2001

51. Winston GP, Yogarajah M, Symms MR, McEvoy AW, Micallef C, Duncan JS: Diffusion tensor imaging tractography to visualize the relationship of the optic radiation to epileptogenic lesions prior to neurosurgery. Epilepsia 52:1430-1438, 2011

52. Zentner J, Hufnagel A, Ostertun B, Wolf HK, Behrens E, Campos MG, et al: Surgical treatment of extratemporal epilepsy: clinical, radiologic, and histopathologic findings in 60 patients. Epilepsia 37:1072-1080, 1996

Manuscript submitted November 25, 2012.

Accepted February 14, 2013.

Please include this information when citing this paper: DOI: 10.3171/2013.2.FOCUS12397.

Address correspondence to: Björn Sommer, M.D., Department of Neurosurgery, University Hospital Erlangen, Schwabachanlage 6, Erlangen 91054, Germany. email: bjoern.sommer@uk-erlangen.de. 